\title{
Treatment of Older Women With Alcohol Problems: Meeting the Challenge for a Special Population
}

\author{
Frederic C. Blow
}

\begin{abstract}
As a larger proportion of the U.S. population reaches late life, there are new challenges to providing quality health care services for this group. Record numbers of adults over 60 are seeking health care for acute and chronic conditions. Older women represent the largest single group of health care users in this country. Twelve percent of older women regularly drink in excess of recommended guidelines (no more than one drink per day or seven drinks per week) and can be considered at-risk drinkers. Problems related to alcohol use and misuse can seriously affect many of the health concerns common among older women, including chronic illnesses and depression. Older women have specific risks and vulnerabilities to alcohol use, which include a swifter progression to alcohol-related illness. However, women in later life who have alcohol problems are underscreened and underdiagnosed, have significant barriers in accessing health care, and respond differentially to standard specialized treatment protocols. To date, research on these topics has been limited. Furthermore, there is a paucity of research focused on treatment outcomes for elderly adults with alcohol problems, with almost no emphasis on women. This paper presents the state of knowledge about alcohol health services for older women and provides recommendations for necessary future health services research on this vulnerable population.
\end{abstract}

$\mathbf{A}^{\mathrm{s}}$ S A LARGER proportion of the U.S. population reaches later life, disease prevention and health promotion among older adults are receiving greater attention. Record numbers of older adults are seeking health care for acute and chronic conditions (Adams et al., 1996). In the United States, older adulthood often is defined as beginning at age 65 . However, the definition of older adulthood varies in research studies and reports. For the purposes of this report, age cutoff points for entry into particular studies will be given.

Although women constitute the majority of older adults, their alcohol intervention and treatment challenges are compelling. There is a lack of alcohol research on older adults in general, with even less research focused on problems related to alcohol use in older women (Center for Substance Abuse Treatment, 1998). Because most older women who drink at risk levels do not meet DSM criteria for alcohol abuse or dependence, alcohol use disorders are least likely to be detected and treated in this population

From the Department of Psychiatry, University of Michigan, and Department of Veterans Affairs Medical Center, Ann Arbor, Michigan.

Received for publication September 7, 1999; accepted June 6, 2000.

An earlier version of this paper was presented at the Conference on Women's Health Services research-"Women and Alcohol Problems"sponsored by the National Institute on Alcohol Abuse and Alcoholism, Conference on Women's Health Services Research, November 5-6, 1998.

Reprint requests: Frederic C. Blow, Ph.D., University of Michigan, Department of Psychiatry, Division of Substance Abuse, 400 E. Eisenhower Parkway, Suite A, Ann Arbor, MI 48108; Fax: 734-930-5150; E-mail: fredblow @umich.edu

Copyright (C) 2000 by the Research Society on Alcoholism.
(Adams et al., 1996). In addition, more older women than men live alone, and older women's substance abuse can be difficult to identify (Moore et al., 1989). Older women with alcohol problems often conceal their drinking or medication/drug misuse because they experience greater stigma associated with use than do men (Gomberg, 1987, 1995).

Alcohol use that increases the risk of consequences from drinking is at-risk drinking. National Institute of Alcohol Abuse and Alcoholism guidelines recommend that older men and women drink no more than seven drinks per week, no more than one drink per day (DuFour and Fuller, 1995; National Institute on Alcohol Abuse and Alcoholism, 1995). Some older adults who drink even small amounts of alcohol may experience alcohol-related problems (e.g., insulin-dependent diabetes, concurrent use of benzodiazepines, mild to moderate cognitive problems). Therefore, both women and men age 65 and over who drink more than seven drinks per week-one per day-can be considered at-risk drinkers. Although they currently may not have a health, social, or emotional problem caused by alcohol, they may experience family, social, or minor health problems, and if this drinking pattern continues over time, these problems could become exacerbated. Women are more likely to decrease alcohol use as the result of brief alcohol interventions (Fleming et al., 1997, 2000) targeted to at-risk drinkers.

Older women who engage in problem drinking are drinking at a level that already has resulted in adverse medical, psychological, or social consequences. Potential consequences can include accidents and injuries, medication interaction problems, and family problems, among others 
(Adams, 1997; Barry and Fleming, 1994; Friedmann et al., 1998).

Compared with men, women have less insurance coverage. In fact, women have a lower level of coverage and fewer women have coverage at all (Estes, 1995). Older women are less likely to have worked and thus are more likely to lose insurance coverage with the death of a spouse. Women drink less often in public places and, therefore, are less likely to drive while intoxicated or engage in other behaviors that might reveal an alcohol problem (Waller et al., 1997). Overall, elderly women are healthier and more independent than men in that age group but are also more isolated. They often drink alone. In addition, older women are prescribed more and consume more psychoactive drugs, particularly benzodiazepines, than do men and are more likely to be long-term users of these substances (Gomberg, 1995).

As part of a 2-year analysis, the National Center on Addiction and Substance Abuse (CASA) at Columbia University (National Center on Addiction and Substance Abuse, 1998) conducted one of the few national surveys that specifically addressed alcohol problems in older women. The study included a nationally representative survey of 400 primary care physicians, at least $10 \%$ of whose patients were women over 59 . Less than $1 \%$ of these primary care physicians even considered a substance abuse diagnosis when presented with the typical early symptoms of alcohol and prescription drug abuse in mature women.

Because of increased health care needs, older adults are more likely than younger adults to seek services from their primary and specialty care providers, and older women are more likely to seek health care than are older men (Fleming and Barry, 1992). This provides the opportunity for health care "gatekeepers" to identify and refer older women who drink at hazardous levels (Center for Substance Abuse Treatment, 1998). As the large number of adults in the Baby Boom generation reach older adulthood, the need for new systematized alcohol screening and brief intervention and treatment techniques targeted to older women will be even more critical to the national health services research agenda.

The introduction of elder-specific alcohol screening instruments such as the Michigan Alcoholism Screening Test-Geriatric version (MAST-G; Blow et al., 1992) and the development of brief alcohol interventions for older adults (Blow et al., unpublished data, 1995-1999; Fleming et al., 2000) that are particularly successful in reducing alcohol use in older women make it possible to focus innovative approaches targeted to this vulnerable underrecognized population.

\section{EXTENT OF THE PROBLEM}

Alcohol Abuse/Dependence in Older Women. Reports of alcohol abuse in older women have varied widely. The CASA study suggested that, of the 25.6 million women over age 59 in the United States, 1.8 million (7\%) abuse alcohol and 2.8 million (11\%) abuse psychoactive drugs (National Center on Addiction and Substance Abuse, 1998). On the other hand, the National Institute of Mental Health Epidemiologic Catchment Area study showed that $<1 \%$ of older women meet criteria for alcohol abuse or dependence (Robins and Reiger, 1991). Older women are more likely to be hospitalized for substance abuse-related problems than for heart attacks (Adams et al., 1993). The substance abuserelated medical problems and injuries resulted in approximately $\$ 30$ billion in health care bills for 1998 (Center for Substance Abuse Treatment, 1998) and may reach $>\$ 100$ billion by the year 2020 given present trends.

Most of the published research on alcohol use disorders in older adults does not focus specifically on women. There is a body of research that links alcohol abuse and dependence in younger women to increased risks for morbidity (Chou and Dawson, 1994) and co-occurring depression (Hesselbrock, 1991; Pettinati et al., 1997). These issues need to be addressed more fully in older women with a range of alcohol problems. Nonetheless, given the emerging knowledge about the consequences to older women who drink above guidelines (Adams et al., 1996; Fleming et al., 2000), previous findings on mixed-gender samples of older adults have relevance for women. Additionally, older women have specific risks and vulnerabilities related to their alcohol use, which include a swifter progression to alcohol-related illness, increased sensitivity to alcohol and psychoactive drugs, more financial issues related to health care coverage, and greater social stigmatization than older men (Center for Substance Abuse Treatment, 1998; National Center on Addiction and Substance Abuse, 1998). Although the problems related to elderly problem drinking provide compelling reasons for a major research focus, the additional susceptibilities of older women who drink above guidelines make them a critical population. Research is needed to determine the best practices for alcohol interventions and to determine better ways to educate professionals who will be expected to recognize, intervene with, and treat these women who are at risk or have developed consequences related to their drinking.

Alcohol use disorders are important public health problems in older adults. Heavy alcohol use is associated with a number of adverse health effects in this population. These include greater risk for harmful drug interactions, injury from accidents, depression, memory problems, liver disease, cardiovascular disease, cognitive changes, and sleep problems (Finch and Barry, 1992; Gambert and Katsoyannis, 1995; Liberto et al., 1992). Older women, because of their increased sensitivity to alcohol and the potential "telescoping" of symptoms (experiencing more severe drinking consequences and symptoms in a relatively short period of time) (Gomberg, 1995), are particularly at risk for these alcohol-related health consequences.

Clinical models of alcoholism and recovery traditionally were thought to follow a natural progression from early 
signs and symptoms through end-stage disease (Fleming and Barry, 1992). When patients "hit bottom" they either died or began the long road to recovery. However, it is not clear that there is a single course to this illness, and many alcohol-dependent people have periods of abstinence or low-risk use. Variations in patterns of use for persons with alcohol problems include those of the early-onset problem drinker who uses alcohol heavily throughout most of adulthood, the cyclical heavy drinker, and the late-onset problem drinker. Late-onset problem drinkers often begin drinking due to stressors of later life (e.g., retirement, death of spouse, diminished physical capacity). Older women are more likely to develop alcohol problems for the first time later in life (Gomberg, 1995; Center for Substance Abuse Treatment, 1998). The implications of their slightly higher rate of late-onset drinking problems are the increased need for focused alcohol and drug screening of older women in health care settings, and the potential for improved response to brief alcohol interventions, as well as greater treatment compliance and enhanced outcomes compared with early onset-problem drinkers and older men in general (Center for Substance Abuse Treatment, 1998).

Prevalence estimates for older male and female at-risk and problem drinkers according to community surveys have ranged from $1 \%$ to $15 \%$ (Adams et al., 1996; Gurland and Cross, 1982; Schuckit and Pastor, 1978). The rates for older women have been suggested to be substantially lower than rates for older men. Estimates for older women generally are reported to be from $<1 \%$ who meet DSM criteria (Bucholz et al., 1995) to $12 \%$ of older women who screen positive for at-risk drinking (Adams et al., 1996) in primary care samples. These rates vary widely depending on the definition of alcohol use disorders and risk drinking as well as the methodology used to obtain samples. Several researchers also have questioned the accuracy of rates of alcohol problems for older adults because the rates were derived by using assessment instruments developed on younger populations. Women in general, and older women in particular, have symptom profiles that have not been well addressed by standard alcohol screening instruments, which therefore leads to the potential for underestimating the extent of the problem in this population.

Older adults seen in medical settings have consistently higher rates of alcohol-related problems (Adams et al., 1993; DuFour and Fuller, 1995) than those in the general population because problem drinkers of all ages are more likely to seek medical care (Beresford, 1979; Institute of Medicine, 1990). Among elderly patients who seek treatment in hospitals, primary care clinics, and nursing homes for medical or psychiatric problems, rates of concurrent alcohol abuse and dependence have been reported between 15\% and 58\% (Adams et al., 1996; Beresford et al. 1990; Buchsbaum et al., 1991; Schuckit, 1982) with older women at the lower end of that range. Although the prevalence of alcohol dependence is lower among older adults, in one study of hospital discharge data, the 65 -and-older group consistently had the highest proportion (approximately $60 \%$ ) of alcohol-related diagnoses that were not primary diagnoses (Stinson et al. 1988). This may reflect increased susceptibility to alcohol-related health problems even at levels below abuse or dependence.

\section{Risk Drinking Among Older Women}

Heavy alcohol consumption in older women can be medically, psychologically, and socially hazardous even if the frequency and quantity of drinking do not warrant a formal diagnosis of alcohol abuse or dependence.

As the health care system moves to managed care models, the development of strategies to deal with older women who are at risk because of their level of alcohol consumption is a growing concern. In a large primary care study of 5065 patients over 60, Adams et al. (1996) found that $15 \%$ of the men and $12 \%$ of the women sampled regularly drank in excess of the limits previously recommended by the National Institute of Alcoholism and Alcohol Abuse, $>7$ drinks per week for women and $>14$ drinks per week for men; guidelines now recommend no more than one drink a day for both men and women over 65 . These guidelines are consistent with empirical evidence for risk-free drinking among older adults (Chermack et al., 1996). Nine percent of the men and $2 \%$ of the women in their sample regularly consumed $>21$ drinks/week. Consistent with findings in younger populations, the study showed that fewer older women than men drink over recommended limits, but there is still a substantial percentage nationwide who are at risk. Therefore, older women who drink in excess of recommended limits and seek primary health care represent an important group for targeted early screening and brief interventions. In addition, a proportion of older women who drink may be affected adversely by even low levels of alcohol consumption. This is a particularly important group because they do not meet cutoffs for risk drinking but have problems due to alcohol/medication interactions and chronic illnesses. For this group, even low levels of drinking can increase the risk of injury and/or health problems and may occur in a large proportion of older women who come into contact with health care professionals (Cyr and Wartman 1988; Moore et al. 1989).

\section{Misuse of Psychoactive Prescription Drugs}

Although the risks of alcohol misuse are substantial, use of psychoactive prescription drugs is most common among white, older women (National Center on Addiction and Substance Abuse, 1998). Relatively little is known about older women who are dependent on psychoactive medications. However, psychoactive medications are the leading cause of hospitalizations due to adverse drug interactions, and the benzodiazepines are among the medications that most often result in adverse consequences (U.S. General Accounting Office, 1995). More research is needed on the nature and extent of psychoactive substance abuse, partic- 
ularly anxiolytics and sedative/hypnotics, in older women (Center for Substance Abuse Treatment, 1998).

\section{BARRIERS TO IDENTIFICATION AND TREATMENT}

Both the detection of drinking problems and provision of appropriate interventions are particularly problematic for older women. Multiple barriers make intervention and treatment less likely in this population. These include shame and guilt, lack of recognition by health care providers, and lack of mobility and transportation difficulties.

\section{Shame and Guilt}

Early in the 20th century, drinking and drunkenness were better tolerated in men, with more taboos and restrictions on the use and misuse of alcohol for women. These taboos, although loosened, have continued to the present. Older women developed their attitudes and behaviors toward alcohol during a period of greater social restrictions on drinking by women. Thus, many older women have shame and guilt associated with excessive drinking and are more likely to hide their drinking, which makes it even more difficult for health care professionals and others, including women themselves, to recognize their alcohol-related problems.

\section{Lack of Recognition by Health Care Providers}

Symptoms of problem drinking often are less visible among older women because they can be masked by other medical, social, or psychological conditions as well as by health care providers' assumptions that older women do not drink. In addition, sensitivity and tolerance to ethanol may be affected by physiological aging processes, as well as by health conditions common to old age (Fleming and Barry, 1992). Older women are significantly more vulnerable to the effects of alcohol than are older men, and older women are more likely to use alcohol in combination with prescribed psychoactive drugs (National Center on Addiction and Substance Abuse, 1998). To reiterate, many health problems seen among older women, such as poor nutrition and polypharmacy, may be exacerbated even when small amounts of alcohol are consumed (Vestal et al., 1977). What might be considered light or moderate drinking for individuals in their 30s may have significant untoward health effects in an older person. Although health care settings are ideal for identifying older women with drinking problems, these issues, combined with stereotyped views of older women, present significant barriers to the identification and treatment of alcohol problems.

\section{Lack of Mobility and Transportation Issues}

The barriers that prevent identification and treatment of alcohol problems among all older females are even greater for those who are homebound $(>70 \%$ of home care pa- tients are women; Dey, 1996) or have trouble with mobility. These women are less likely to have the social and health care contacts that enable identification of alcohol problems.

Transportation difficulties can limit social and health care-seeking contacts, alcohol treatment seeking if a problem is detected, and aftercare follow-up if initial alcohol treatment is provided. This is especially problematic for older women who live in rural communities that lack public transportation or in poor urban communities where accessing transportation can be dangerous.

\section{ALCOHOL TREATMENT RESEARCH ON OLDER WOMEN}

There have been few systematic studies of alcoholism treatment outcomes among older adults and even fewer that included women (Atkinson, 1995; Gomberg, 1988). Alcohol treatment research that includes any older women generally focuses on alcohol abuse and dependence. The majority of older women with alcohol-related problems drink at risky levels but do not meet criteria for abuse and dependence. There is one published study (Fleming et al., 2000) and ongoing work (Blow et al., National Institute on Alcohol Abuse and Alcoholism Grant 2 P50 AA07378) to date focused specifically on alcohol interventions for older women who do not meet DSM-IV abuse/dependence criteria.

The study of treatment outcomes for older women who meet criteria for alcohol abuse/dependence has become a critical issue because of their unique needs, which include stigma, insurance and financial considerations, and telescoping of symptoms. Because traditional residential alcoholism treatment programs generally provide services to very few older women, sample sizes have been inadequate to study treatment outcomes among elderly women who meet criteria for alcohol abuse and dependence. The development of elder-specific alcoholism treatment programs in recent years may facilitate studies of this special population (Atkinson, 1995).

Previous research on elderly alcoholism treatment can be placed into two broad categories: treatment compliance studies and prospective studies of treatment outcomes. Also, most studies of older persons in alcohol treatment have been conducted in Department of Veterans Affairs (VA) settings, which thus limits inclusion of women.

\section{Treatment Compliance}

Most compliance studies have been conducted with older men. Older persons with alcohol disorders were significantly more likely to complete treatment than younger patients (Schuckit 1977; Wiens et al., 1982-83). Atkinson et al. (1993) also found that the proportion of older male alcoholics who completed treatment was twice that of younger men. Despite generally better compliance rates, two studies have shown that programming which addressed problems and concerns specific to older adults in treatment 
groups separate from younger adults (age-specific programming) improved treatment completion and resulted in higher rates of attendance at group meetings (Atkinson, 1995; Kofoed et al., 1987).

Much of the treatment compliance research with older adults has focused on age of onset as a major variable of interest. The research studies have not used consistent definitions of early versus late onset. Schonfeld and Dupree (1991) used a matched-pairs post hoc design to examine the rates of completion of 6-month day treatment for a small sample of older men and women alcoholics $(n=23)$ who were age 55 and older. Although the sample size was small, the results provided a basis for studying a little-addressed treatment issue. The older adults whose problem drinking began before age 50 (early onset) were compared with those who began problem drinking after age 50 (late onset). Those classified as late-onset alcoholics were significantly more likely to complete treatment. However, in a subsequent report that included the larger sample of 148 from which these patients were selected, there was no difference in completion rate based on age of onset (Schonfeld and Dupree 1991). Further research in this area is needed to determine if age of onset predicts treatment outcome for older adults and the best practices for the treatment of both early- and late-onset alcohol problems.

Atkinson and colleagues (1990) conducted a study that focused on male alcoholic veterans age 60 and older in which the sample was divided into early-onset (alcoholism beginning at age 40 and younger, $n=50$ ), midlife-onset $(41-59, n=62)$, and late-onset (age 60 and older, $n=20$ ) subgroups. Age of problem drinking onset was related to program completion and to weekly meeting attendance, with the late-onset subgroup showing the best compliance. A subsequent study of 128 older men in alcoholism treatment (age 55 and older) found that drinking relapses during treatment were unrelated to age of onset (Atkinson et al., 1993). Studies on the impact of age of onset on treatment compliance have yielded mixed results with older males. Because the work in the area of onset and treatment compliance generally has targeted men, and because more older women than men have late onset of the illness and telescoping of symptoms, more work is needed to understand the relationship between age of onset and treatment compliance among older women.

\section{Prospective Studies of Treatment Outcomes}

Few prospective treatment outcome studies are reported in the literature. Most published studies have targeted older men rather than women. Sample sizes of men tend to be too small to provide definitive results. In one study, 137 male veterans (age 45-59 years, $n=64$; age 60-69 years, $n=62$; age 70 years and older, $n=11$ ) with alcohol problems were randomly assigned after detoxification to age-specific treatment or standard mixed-age treatment (Kashner et al., 1992). Those patients in the elder-specific program were 2.9 times more likely at 6 months and 2.1 times more likely at 1 year to report abstinence compared with mixed-age group patients. Unfortunately, baseline alcohol consumption and alcohol severity data were not included in the study, so conclusions related to changes in consumption and severity could not be drawn.

Because older women are less likely to seek alcohol treatment due to personal barriers (e.g., financing, stigma) and health care barriers (e.g., providers who do not easily recognize alcohol problems in older women), there are fewer women than men in treatment. Therefore, there are even fewer opportunities to study women in treatment and, due to sample sizes needed for conclusive results, women often have been excluded from studies.

In a study that addressed both sexes, Rice and colleagues (1993) compared drinking outcomes for randomly assigned men and women alcoholics 3 months after being matched to one of three age-mixed outpatient treatment conditions. Older patients treated in an individual-focused condition had the greatest number of abstinent days and the fewest number of heavy drinking days when compared with older patients in a group treatment condition. This study suggested that elderly men and women with alcohol disorders may respond better to individual-focused interventions rather than traditional mixed-age group-oriented treatment.

\section{Brief Alcohol Interventions}

A number of randomized controlled trials in other countries have demonstrated that brief advice therapy can reduce alcohol use and related problems in at-risk or nondependent problem drinkers (Anderson and Scott, 1992; Fleming et al., 1997; Kristenson et al., 1983; Persson and Magnusson, 1989; Saunders et al., 1993; Wallace et al., 1988). However, most of the brief intervention trials have excluded individuals age 65 and older. Because older adults, and older women in particular, with problems related to their drinking generally do not fit into the abuse/ dependence definition, new work is beginning to focus on at-risk drinking in this population. More research is needed in this area.

Only two randomized controlled trials have focused on older male and female problem drinkers. In a study of brief physician advice with at-risk drinkers aged 65 and over, Project GOAL (Guiding Older Adult Lifestyles) found positive results in the changes in drinking patterns of the experimental $(n=158)$ versus the control group $(n=71)$. At the time of the 12-month follow-up, there was a significant reduction in 7-day alcohol use, episodes of binge drinking, and frequency of excessive drinking (Fleming et al., 2000). Women were more likely than men in this sample to reduce their drinking and follow recommended guidelines. The results indicated that brief physician advice made a difference in the drinking patterns of older at-risk and problem drinkers. 
Blow and colleagues currently are completing a 5-year study of an elder-specific alcohol brief intervention for older drinking adults screened in primary care settings. The elder-specific intervention contains both brief advice by a psychologist or social worker and motivational interviewing techniques (Miller and Rollnick, 1991). The brief intervention was a computer-generated, customized report on selfreported drinking compared with age-specific national drinking patterns that was modeled on the World Health Organization format (Babor and Grant, 1992). A total of 460 older adults ( $25 \%$ women) have been randomized in this ongoing trial; $>26 \%$ were African Americans. Preliminary results from this study suggest that both women and men benefit from the elder-specific intervention in terms of reduced alcohol consumption and binge drinking compared with the control group. Women in this study were more likely to change drinking behavior simply as a result of screening for alcohol problems than were men. Future studies that focus on a variety of brief intervention techniques as well as on screening systems will help optimize outcomes for older women at-risk drinkers.

\section{Limitations of Treatment Outcome Research}

There remain major limitations in the treatment compliance literature for older adults, which include a significant lack of women in the studies, lack of drinking outcome data, failure to report treatment dropouts, and variations in definitions of treatment completion. Standardized definitions are necessary to adequately compare study results in this growing field of research.

Few carefully controlled, prospective treatment outcome studies that include sufficiently large numbers of older adults with alcoholism have been conducted to address some of the limitations of previous research (e.g., sample size and inadequate statistical power, lack of control group). These studies include few women, which decreases the generalizability of their results.

In addition, age cutoffs for inclusion have varied widely and have included nonelderly individuals in the "older" category, with several studies including individuals as young as age 45. Furthermore, the majority of studies have used relatively unstructured techniques for assessing alcoholrelated symptoms and consequences of drinking behavior. Finally, the manner in which outcomes have been assessed has been narrow in focus. Most studies have dichotomized treatment outcome (abstention versus relapse) based solely on drinking behavior.

\section{COST AND REIMBURSEMENT ISSUES}

Because older women comprise the majority of the older adult population and often have fewer financial resources, including supplemental health insurance, specialized alcohol treatment programs may be less available to them (Center for Substance Abuse Treatment, 1998). Therefore, cost and reimbursement issues are of particular importance in the context of treating older women with drinking problems. Most of the alcohol-related treatment cost data available today do not focus on older adults in general or on women specifically. However, the available data can be extrapolated cautiously to seniors.

In addition, with ongoing changes in the delivery of alcohol treatment services from inpatient to outpatient settings coupled with the shifting reimbursement structure from fee-for-service Medicare to managed Medicare, we can anticipate changes in treatment options for older adults. These changes in treatment venue and fee structures offer the opportunity for conducting multidimensional outcomes assessments in the context of various types of quality management efforts.

\section{Cost Issues for Alcohol Treatment}

Older adults are high users of health care, and older substance abusers use substantial amounts of care (Adams et al., 1993; National Center on Addiction and Substance Abuse, 1998). Of all Medicare hospital admissions for older women, $8.3 \%$ are attributable to substance abuse (National Center on Addiction and Substance Abuse, 1998). Research needs to be conducted on the percentage of older women who have hospital admissions related to substance abuse. It will be important to determine comorbidities, treatment options, and costs in this area to determine the impact of substance abuse in later life on the potential cost-effectiveness of varying interventions. For the purposes of this article, a brief review of treatment cost studies in general populations is presented to highlight the potential issues relevant to older women.

Persons with alcohol dependence consume $>15 \%$ of the national health care budget (Rice et al., 1993). However, only about $10 \%$ of the total cost of alcoholism to society is spent on clinical care costs (Rice et al., 1993). The total costs of alcoholism to society are estimated at $>\$ 100$ billion per year. The cost is so high because alcohol misuse and abuse can lead to increased mortality, significant social costs, and health consequences (Brower et al., 1994; Goodman et al., 1991; Holder and Blose, 1992; National Institute on Alcohol Abuse and Alcoholism, 1995).

A number of cost studies are directed to younger adults (Finney and Monahan, 1996; Holder and Blose, 1992; Holder et al., 1991). Few studies separate out the costs of alcohol disorders for older adults or even include older adults in cost analyses. Older women generally have not been studied as a separate category.

With the emergence of prevention and intervention strategies in managed care settings, coupled with the proliferation of managed Medicare programs, more economic analyses need to be conducted on the cost-effectiveness of alcohol services, which include primary prevention and brief intervention strategies provided to enrollees. One of the few recent studies of managed care (Holder et al., 1995) estimated that for every $\$ 10,000$ spent on brief interven- 
tions for alcohol or drug abuse, $\$ 13,500$ to $\$ 25,000$ is saved in medical spending for the managed care provider. Filling in the gaps is important, particularly regarding the growing population of older women, because managed care providers are challenged to provide necessary services at lower cost.

Caution in reviewing the results and cost-effectiveness of brief interventions has been suggested (Heather, 1995; Peele, 1990). Among other issues, these reviews generally do not assess the costs and needs of older women in these settings. Comparisons are difficult because cost analysis studies do not use consistent methodologies. There are problems in interpreting the data from brief intervention studies because brief interventions are not a homogeneous entity. They vary in length, structure, targets of intervention, and personnel responsible for delivery, and there is a distinction between treatment seekers (i.e., persons who answer ads and indicate that they would like to decrease their drinking) and nontreatment seekers (i.e., individuals with regularly scheduled appointments for medical problems who receive interventions from their health care providers) (Heather, 1995). Clear delineation of the type of study and the potential audience for the research can help to alleviate problems of misinterpretation.

\section{Reimbursement Issues in the Treatment of Older Adults}

Reimbursement mechanisms for specialized alcohol treatment in this population remain one of the important challenges. The reimbursement systems have not been adapted to the serious needs of older women with alcohol problems. These individuals often experience longer, more severe withdrawal and have more related medical problems, which increases the need for inpatient treatment in an era when inpatient treatment options are disappearing. Acute inpatient care may be particularly important for older women because of the increased likelihood of having more physical and cognitive problems that may preclude participation in group programming in the early stages of treatment. These women may need a more intensive outpatient experience after any inpatient stay than younger adults. Finally, mechanisms to reimburse alcohol prevention and early intervention efforts in primary care settings are needed, because research indicates that early intervention programs are effective with a large proportion of older women who are at-risk and problem drinkers. These initiatives will provide cost savings to the medical community by preventing more costly complications of heavier alcohol intake.

The structure of insurance policies also can be a barrier to treatment. The "carving out" of mental health services from physical health services under managed care in particular can prevent older women from receiving alcohol treatment services. Continuity of care for older women may be enhanced by integrating alcohol services into health plans rather than by carving out these services. Because older women have specific problems with transportation, financing, and stigma, the seamless provision of alcohol treatment, including brief intervention strategies, could maximize access, compliance, and outcomes of care.

\section{OLDER WOMEN AND ALCOHOL: CRITICAL ISSUES FOR FUTURE RESEARCH}

Given the rapidly expanding older female segment of the population and the seriousness of alcohol problems in older women, the literature on alcohol problems among older women is surprisingly sketchy. "Substance-abusing older adults" as a distinct definable subgroup was first described in the research literature in 1964 (Droller, 1964). Researchers in the 1960s and 1970s were convinced that alcohol abuse was rare among older adults, in general (Atkinson and Ganzini, 1994), and almost nonexistent in older women. Thus, researchers and clinicians alike widely believed that alcohol use disorders among this age group did not merit the attention given to drinking and drug abuse among younger adults (Bucholz et al., 1995).

There is evidence that alcohol-related problems in older men and women are significant and increasing public health concerns (American Medical Association Council on Scientific Affairs, 1996; Beresford, 1995; Liberto et al., 1992). Because of the relative frequency and social acceptance of regular alcohol use among the "Baby Boom" segment of the population, it is likely that as these individuals reach old age, the prevalence of alcohol problems in late life will increase (Atkinson, 1995; Liberto et al., 1992). Harmful effects of drinking among elderly women are experienced at lower consumption levels than younger drinkers, and alcohol abuse and dependence can exacerbate problems with physical and mental health functioning associated with aging (Atkinson, 1995; Liberto et al., 1992). Older adults who enter alcohol treatment may have unique psychosocial and health needs that may be related significantly to treatment needs and outcome (Gomberg, 1995).

The underrepresentation of older women in traditional mixed-age programs and the scarcity of specialized, agespecific programs have contributed to the paucity of research on treatment outcome for the older adult treatment population (Atkinson, 1995). Previous research on alcohol problems in the elderly has focused almost exclusively on men. Notable exceptions have been more recent studies in primary care settings (Adams et al., 1996; Fleming et al., 2000). The recruitment of older women with alcohol misuse, abuse, and dependence presents real challenges because fewer older women are problem drinkers and even fewer enter treatment settings. In addition, because many older women experience strong feelings of shame or guilt regarding their drinking, it is often difficult to recruit them into studies even if they have been identified as having alcohol problems. 


\section{Key Research Issues}

Four key issues need to be addressed in future research on the treatment of alcohol problems in older women: (1) the multiple problems (e.g., prescription drug abuse, use of tobacco, depression, anxiety, comorbid chronic physical conditions) faced by older women with alcohol problems; (2) the feasibility and development/integration of genderspecific alcohol services for older women and alternative models of care; (3) the impact of policy changes on older women in terms of treatment effectiveness, outcomes, access and cost of services on national, state, and local levels; and (4) the development of age- and gender-appropriate assessment and outcome measures.

First, from a clinical perspective, alcohol problems in older women generally are not seen in isolation. Older women with alcohol problems often present a complex clinical picture. To appropriately target alcohol interventions in this population, research needs to be conducted that will describe, understand, and predict outcomes for older women with a range of physical and mental health issues that include their use of alcohol and other psychoactive substances. To adequately address the concerns specific to alcohol problems in older women, more research is needed on the misuse of psychoactive prescription medications, interactions of medications and alcohol, depression and anxiety as warning signs of substance abuse, cooccurring physical illnesses, the role of alcohol and drugs in cognitive impairments, issues of family support and potential elder abuse, and the cultural context of drinking and abstinence. These studies are needed in varying health care settings including primary care, hospitals, and home health care delivery systems. A focus on care patterns and intervention/treatment alternatives with a variety of health care providers (e.g., nurses, social workers, primary care physicians, home health care workers) will enhance training and service delivery options for older women whose use of alcohol or drugs puts them at risk for a variety of health complications.

Second, the development and testing of gender-sensitive screening, brief intervention strategies, and treatment services for older women should be a priority. The settings for these studies could be in primary care, home health services, and elder congregate locations (e.g., housing, senior centers, retirement communities). Additional studies are needed to determine which strategies and types of services are the most effective for this special population. As described previously, future research will need to attend to barriers to engagement in the treatment process and access to treatment services.

Third, as more older adults enroll in managed Medicare programs, there is likely to be continued pressure to limit coverage of substance abuse service. Limiting coverage could have a particularly detrimental effect on older women because they will have even less access to care and, consequently, may experience more complications related to their continued drinking. Research on the implications of Medicare policy changes is needed to determine whether the level of copay, restrictions on service availability, or restrictions on type of specialty care will affect outcomes.

Research that develops and uses appropriate assessment and outcome measures for older women is imperative. As discussed earlier, there has been limited use of standardized assessment and outcome measures in the few published alcohol treatment research studies with older adults. Before conducting new treatment outcome research in this area, researchers need to develop and test age- and gendersensitive instruments that assess the process and outcomes of alcohol treatments for older women.

Finally, with the rapidly changing health care environment, it is only through new research focused on optimal approaches to identification, diagnosis, intervention, treatment, and organization of care that we can meet the challenge of safeguarding the health of at-risk older women.

\section{REFERENCES}

Adams WL (1997) Interactions between alcohol and other drugs, in Older Adults' Misuse of Alcohol, Medicines, and Other Drugs: Research and Practice Issues (Gurnack AM ed), pp. 185-205. Springer, New York.

Adams WL, Barry KL, Fleming MF (1996) Screening for problem drinking in older primary care patients. JAMA 276:1964-1967.

Adams WL, Yuan Z, Barboriak JJ, Rimm AA (1993) Alcohol-related hospitalizations in elderly people. JAMA 270:1222-1225.

American Medical Association, Council on Scientific Affairs (1996) Alcoholism in the elderly. JAMA 275:797-801.

Anderson P, Scott E (1992) The effect of general practitioners' advice to heavy drinking men. Br J Addict 87:891-900.

Atkinson RM (1995) Treatment programs for aging alcoholics, in Alcohol and Aging (Beresford T, Gomberg E eds), pp 186-210. Oxford University Press, New York.

Atkinson RM, Ganzini L (1994) Substance abuse, in Textbook of Geriatric Neuropsychiatry (Coffey CE, Cummings JL eds), pp 297-321. American Psychiatric Press, Washington, DC.

Atkinson RM, Tolson RL, Turner JA (1990) Late versus early onset problem drinking in older men. Alcohol Clin Exp Res 14:574-579.

Atkinson RM, Tolson R, Turner JA (1993) Factors affecting outpatient treatment compliance of older male problem drinkers. J Stud Alcohol 54:102-106.

Babor TF, Grant M (eds) (1992) Project on Identification and Management of Alcohol-Related Problems. Report on Phase II: A Randomized Clinical Trial of Brief Interventions in Primary Health Care. World Health Organization, Geneva, Switzerland.

Barry KL, Fleming MF (1994) The family physician. Alcohol Res Health 18:105-109.

Beresford TP (1979) Alcoholism consultation and general hospital psychiatry. Gen Hosp Psychiatry 1:293-300.

Beresford TP (1995) Alcoholic elderly: Prevalence, screening, diagnosis, and prognosis, in Alcohol and Aging (Beresford TP, Gomberg E eds), pp 3-41. Oxford University Press, New York.

Beresford TP, Blow FC, Hill E, Singer K, Lucey MR (1990) Comparison of CAGE questionnaire and computer-assisted laboratory profiles in screening for covert alcoholism. Lancet 336:482-485.

Blow FC, Brower KJ, Schulenberg JE, Demo-Dananberg LM, Young JP, Beresford TP (1992) The Michigan Alcoholism Screening Test-Geriatric Version (MAST-G): A new elderly-specific screening instrument Alcohol Clin Exp Res 16:372.

Brower KJ, Mudd S, Blow FC, Young JP, Hill EM (1994) Severity and treatment of alcohol withdrawal in elderly versus younger patients. Alcohol Clin Exp Res 18:196-201. 
Bucholz KK, Sheline Y, Helzer JE (1995) The epidemiology of alcohol use, problems, and dependence in elders: A review, in Alcohol and Aging (Beresford TP, Gomberg E eds), pp 19-41. Oxford University Press, New York

Buchsbaum DG, Buchanan RG, Centor RM, Schnoll SH, Lawton MJ (1991) Screening for alcohol abuse using CAGE scores and likelihood ratios. Ann Intern Med 115:774-777.

Center for Substance Abuse Treatment (1998) Treatment Improvement Protocol \#26: Substance Abuse Among Older Adults. Frederic C. Blow, Chair Substance Abuse and Mental Health Services Administration, DHHS No. (SMA)98-3179. Center for Substance Abuse Treatment, Rockville, MD.

Chermack ST, Blow FC, Hill EM, Mudd SA (1996) The relationship between alcohol symptoms and consumption among older drinkers. Alcohol Clin Exp Res 20:1153-1158.

Chou SP, Dawson DA (1994) A study of the gender differences in morbidity among individuals diagnosed with alcohol abuse and/or dependence. J Subst Abuse 6:381-394.

Cyr MG, Wartman SA (1988) The effectiveness of routine screening questions in the detection of alcoholism. JAMA 259:51-54.

Dey AN (1996) Characteristics of Elderly Home Health Care Users: Data from the 1994 National Home and Hospice Care Survey. Advance Data From Vital and Health Statistics, No 279. National Center for Health Statistics, Hyattsville, MD.

Droller H (1964) Some aspects of alcoholism in the elderly. Lancet 1:137-139.

DuFour M, Fuller R (1995) Alcohol in the elderly. Annu Rev Med 46:123-132.

Estes CL (1995) Mental health services for the elderly: Key policy elements, in Emerging Issues in Mental Health and Aging (Gatz M ed), pp 301-318. American Psychological Association, Washington, DC.

Finch J, Barry KL (1992) Substance use in older adults, in Addictive Disorders (Fleming M, Barry K eds), pp 270-286. Mosby/Yearbook Medical Publishers, St. Louis, MO.

Finney JW, Monahan SC (1996) The cost effectiveness of treatment for alcoholism: A second approximation. J Stud Alcohol 57:229-243.

Fleming MF, Barry KL (eds) (1992) Addictive Disorders: A Practical Guide to Treatment. Mosby/Yearbook Medical Publishers, St. Louis, MO.

Fleming MF, Barry KL, Manwell LB, Johnson K, London R (1997) Brief physician advice for problem alcohol drinkers: A randomized controlled trial in community-based primary care practices. JAMA 277:1039-1045.

Fleming M, Manwell LB, Barry KL, Adams W, Stauffacher E (2000) Guiding older adult lifestyles (Project GOAL): The effectiveness of brief physician advice for alcohol problems in older adults. J Fam Pract, in press.

Friedmann PD, Saitz R, Samet JH (1998) Management of adults recovering from alcohol or other drug problems: Relapse prevention in primary care. JAMA 279:1227-1231.

Gambert SR, Katsoyannis KK (1995) Alcohol-related medical disorders of older heavy drinkers, in Alcohol and Aging (Beresford T, Gomberg ESL, eds), pp 70-81. Oxford University Press, New York.

Gomberg ESL (1987) Shame and guilt issues among women alcoholics. Alcohol Treat Q 4:139-155.

Gomberg ESL (1988) Overview: Issues of alcohol use and abuse in the elderly population. Pride Institute J Long Term Home Health Care $7: 4-17$.

Gomberg ESL (1995) Older women and alcohol use and abuse. Recent Dev Alcohol 12:61-79.

Goodman AC, Holder HD, Nishiura E (1991) Alcoholism treatment offset effects: A cost model. Inquiry 28:168-178.

Gurland BJ, Cross PS (1982) Epidemiology of psychopathology in old age: Some implications for clinical services. Psychiatr Clin North Am 5:1126.

Heather N (1995) Interpreting the evidence on brief interventions for excessive drinkers: The need for caution. Alcohol Alcohol 30:287-296.

Hesselbrock MN (1991) Gender comparison of antisocial personality and depression in alcoholism. J Subst Abuse 3:205-219.
Holder HD, Blose JO (1992) The reduction of health care costs associated with alcoholism treatment: A 14 year longitudinal study. J Stud Alcohol 53:293-302.

Holder H, Boyd G, Howard J, Flay B, Voas R, Grossman M (1995) Alcohol-problem prevention research policy: The need for a phases research model. J Public Health Policy 16:324-346.

Holder HD, Longabaugh R, Miller WR, Rubonis AV (1991) The cost effectiveness of treatment for alcoholism: A first approximation. J Stud Alcohol 52:517-540.

Institute of Medicine (1990) Broadening the Base of Treatment for Alcohol Problems. National Academy Press, Washington, DC.

Kashner M, Rodell DE, Ogden SR, Guggenheim FB, Karson CN (1992) Outcomes and costs of two VA inpatient treatment programs for older alcoholic patients. Hosp Community Psychiatry 43:985-989.

Kofoed LL, Tolson RL, Atkinson RM, Toth RL, Turner JA (1987) Treatment compliance of older alcoholics: An elder-specific approach is superior to "mainstreaming." J Stud Alcohol 48:47-51.

Kristenson H, Ohlin H, Hulten-Nosslin M, Trell E, Hood B (1983) Identification and intervention of heavy drinking in middle-aged men: Results and follow-up of 24-60 months of long-term study with randomized controls. Alcohol Clin Exp Res 7:203-209.

Liberto JG, Oslin DW, Ruskin PE (1992) Alcoholism in older persons: A review of the literature. Hosp Community Psychiatry 43:975-984.

Miller WR, Rollnick S (1991) Motivational Interviewing. Guilford Press, New York.

Moore RD, Bone LR, Geller G, Mamon JA, Stokes EJ, Levine DM (1989) Prevalence, detection, and treatment of alcoholism in hospitalized patients. JAMA 261:403-407.

National Center on Addiction and Substance Abuse (1998) Under the Rug: Substance Abuse and the Mature Woman. Columbia University, New York.

National Institute of Alcoholism and Alcohol Abuse (1995) The Physicians' Guide to Helping Patients With Alcohol Problems. NIH Publ No 95-3769. National Institutes of Health, Bethesda, MD.

Peele S (1990) Research issues in assessing addiction treatment efficacy: How cost effective are Alcoholics Anonymous and private treatment centers? Drug Alcohol Depend 25:179-182.

Persson J, Magnusson PH (1989) Early intervention in patients with excessive consumption of alcohol: A controlled study. Alcohol 6:403408.

Pettinati HM, Pierce JD, Wolf AL, Rukstalis MR, O’Brien CP (1997) Gender differences in comorbidly depressed alcohol-dependent outpatients. Alcohol Clin Exp Res 21:1742-1746.

Rice C, Longabaugh R, Beattie M, Noel N (1993) Age group differences in response to treatment for problematic alcohol use. Addiction 88: $1369-1375$.

Robins LN, Reiger DA (1991) Psychiatric Disorders in America: The Epidemiological Catchment Area Study. Free Press, New York.

Saunders JB, Aasland OG, Babor TF, DeLa Fuente JR, Grant M (1993) Development of the Alcohol Use Disorders identification Test (AUDIT): WHO collaborative project on early detection of persons with harmful alcohol consumption-II. Addiction 88:791-804.

Schonfeld L, Dupree LW (1991) Antecedents of drinking for early- and late-onset elderly alcohol abusers. J Stud Alcohol 52:587-592.

Schuckit MA (1977) Geriatric alcoholism and drug abuse. Gerontologist 17:168-174.

Schuckit MA (1982) A clinical review of alcohol, alcoholism, and the elderly patient. J Clin Psychiatry 43:396-399.

Schuckit MA, Pastor PA Jr (1978) The elderly as a unique population. Alcohol Clin Exp Res 2:31-38.

Stinson FS, Dufour MC, Bertolucci D (1988) Alcohol-related morbidity in the aging population. Alcohol Health Res World 13:80-87.

U.S. General Accounting Office (1995) Prescription drugs and the elderly: Many still receive potentially harmful drugs despite recent improvements. U.S. General Accounting Office, Gaithersburg, MD. 
Vestal RE, McGuire EA, Tobin JD, Andreas R, Norris AH, Mezey E (1977) Aging and ethanol metabolism. Clin Pharmacol Ther 231:343354.

Wallace P, Cutler S, Haines A (1988) Randomized controlled trial of general practitioner intervention in patients with excessive alcohol consumption. Br Med J 298:663-668.
Waller PF, Blow FC, Maio RF, Singer K, Hill EM, Schaefer N (1997) Crash characteristics and injuries of victims impaired by alcohol versus illicit drugs. Accid Anal Prev 29:817-827.

Wiens AN, Menustik CE, Miller SL, Schmitz RE (1982-83) Medicalbehavioral treatment of the older alcoholic patient. Am J Drug Alcohol Abuse 9:461-475. 